\title{
Reduction of the Optical Loss in the Multi-Cathode Structure Organic Light Emitting Device Using a Long Range Surface Plasmon
}

\author{
Akiyoshi Mikami \\ Department of Electrical Engineering, Kanazawa Institute of Technology, Ishikawa, Japan \\ Email: mikami@neptune.kanazawa-it.ac.jp
}

Received 9 June 2016; accepted 23 August 2016; published 30 August 2016

\begin{abstract}
Light extraction efficiency of organic light-emitting devices has improved by using a nano-sized multi-cathode structure consisting of semi-transparent metal and an optical compensation layer. From the detail optical calculation based on the multi-scale analysis including near-field optics, it was found that surface plasmon loss in the metal cathode is suppressed to less than $10 \%$ due to long range and short range surface plasmon coupling between both sides of metal cathode. Not less than $90 \%$ of optical power in the dipole emission can be successfully utilized as propagation light. Light extraction efficiency in a phosphorescent device has improved about twice by using the multi-cathode structure.
\end{abstract}

\section{Keywords}

Light-Emitting Device, Organic Material, Surface Plasmon, Optical Simulation

\section{Introduction}

Organic light-emitting devices (OLED) are now widely recognized as a potential application for high quality flat panel displays and general lighting. The internal quantum efficiency of OLED has been achieved near $100 \%$ using phosphorescent materials with proper management of singlet and triplet excitons [1]. However, the external quantum efficiency (EQE) of conventional devices remains $20 \%-25 \%$ because of poor light extraction efficiency. One of the reasons is quite a large losses induced by surface plasmon polariton which is direct interaction between a metal cathode and evanescent wave in near-field of vertical dipole emission [2]. In results, optical energy of propagation wave is restricted only a half of the total emission energy. It is clear that the light outcoupling behavior significantly changes with not only optical constants of materials but also the device structure including substrate, electrode and passivation layers. We have recently found that a multi-cathode (MLC) structure consisting of semi-transparent metal, an optical compensation layer and high reflection metal makes it possible to achieve more than $50 \%$ in out-coupling efficiency by the combination of high refractive index layer 
[3] [4]. The objective of this research is the control of surface plasmon (SP) in OLEDs. This paper will discuss mainly the reason why SP loss can be reduced by employing a nano-sized MLC structure.

\section{Experimental Methods}

\subsection{Device Structure and Sample Preparation}

Figure 1(a) shows a basic device structure of green phosphorescent OLED used in this experiment. The device basically consists of an indium-tin-oxide (ITO) bottom electrode, a Poly(3,4)-ethylendioxy thiophene-polystyrenesulfonate (PEDOT:PSS) hole-injection layer, Bis[(1-naphthyl)-N-phenyl]benzidine (NPB) hole-transporting layer, a 4,4'-N,N'-dicarbazole-biphenyl (CBP) emitting layer (EML) doped with $\operatorname{Ir}(\mathrm{ppy})_{3}$ emitting guest, a 2-(4-Biphenylyl)-5-(4-tert-butylphenyl-1,3,4-oxadiazole) (Bu-PBD) electron transporting layer (ETL) and MgAg cathode. Figure 1(b) shows a proposed new device with MLC structure, which is the feature to havea semitransparent MgAg layer and an optical compensation (OC) layer. Transmittance of OC-layer is more than 90\%, and its refractive index was 1.86.A silver metal on the backside is not an electrode, but a high reflection mirror layer. PEDOT:PSS was formed by spin-coating method and other all layers were deposited by physical deposition process.

\subsection{Optical Analysis}

As well known, an optical energy in OLED is generally divided into a propagation wave and an evanescent wave. The former consists of external, substrate and waveguide modes, and the latter is direct coupling of near-field with surface plasmon polariton and lossy surface waves on the metal cathode. These optical modes can be drawn by using in-plane wave-vector $k_{h}$ as shown in Figure 1(c), where $k_{h}$ and $k_{z}$ are the components of wave-vector $\mathrm{k}_{1}$ parallel and vertical to the interface, respectively. In general, an external mode will be treated by classical ray-optics. In the case of substrate mode, we have to take into account a multiple internal reflection in the thick layer. So the wave-optics of incoherent light will be applied to the mode analysis. Waveguide mode should be calculated by electro-magnetic optics of coherent light. Finally, SP is directly related to the near-field optics. Wide range of optics is deeply involved in theoptical phenomena of OLEDs, so we used multi-scale optical analysis for the optimization of multi-stacked OLED structure. An optical power spectrum, which is defined as an optical energy density as a function of in-plane wave-vector in the dipole emission including near-field and far-field optics was calculated by using original simulation software based on the electromagnetic interaction of molecules with metal interface [5]. For the enhancement of light extraction, the way of using a substrate propagation mode is much practical to take the light out of the device by using high refractive index substrate attached with micro-lens array. However, the sum of external and substrate modes is only less than half of total optical

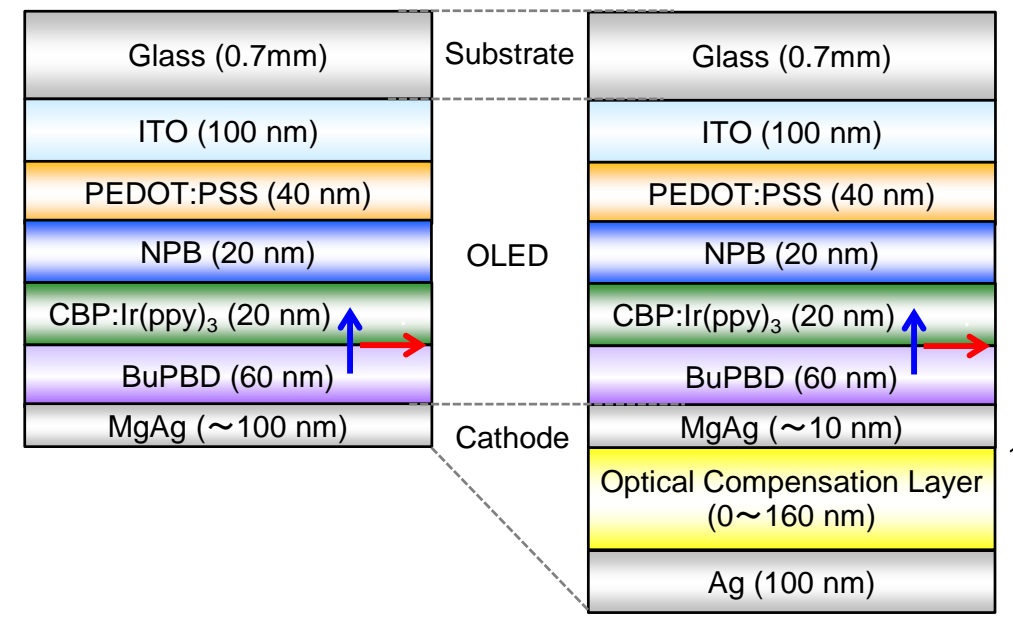

(a) Normal structure

(b) Multi-cathode structure

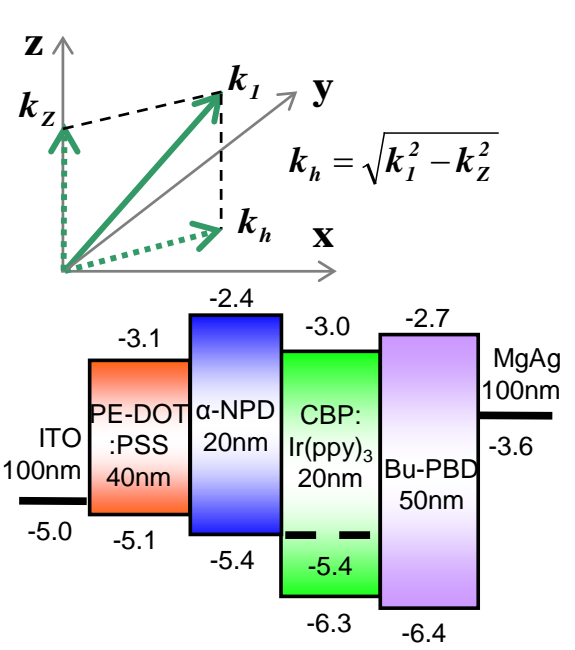

(c) Potential Energy \& Wave Vector

Figure 1. Device structures of phosphorescent green OLED with (a) normal and (b) multi-cathode structure; (c) is potential energy levels of normal structure and definition of wave-vector in the optical device. 
energy even if the device is carefully designed because of large losses induced by waveguide lights and SP. To solve this problem, it is required to suppress the SP loss and then to convert electromagnetic energy propagating as a waveguide mode into the substrate mode. Reducing SP-loss is very important issue in the optical design of high efficiency OLEDs.

\section{Experimental Results}

\subsection{Surface Plasmon Lossat MgAg Cathode}

Figures 2(a)-(c) show a dissipated optical power density as a function of in-plane wave-vector $\left(\mathrm{k}_{\mathrm{h}}\right)$, which is the so-called power spectrum of electric dipole radiation, in phosphorescent green OLEDs with MgAg cathode of
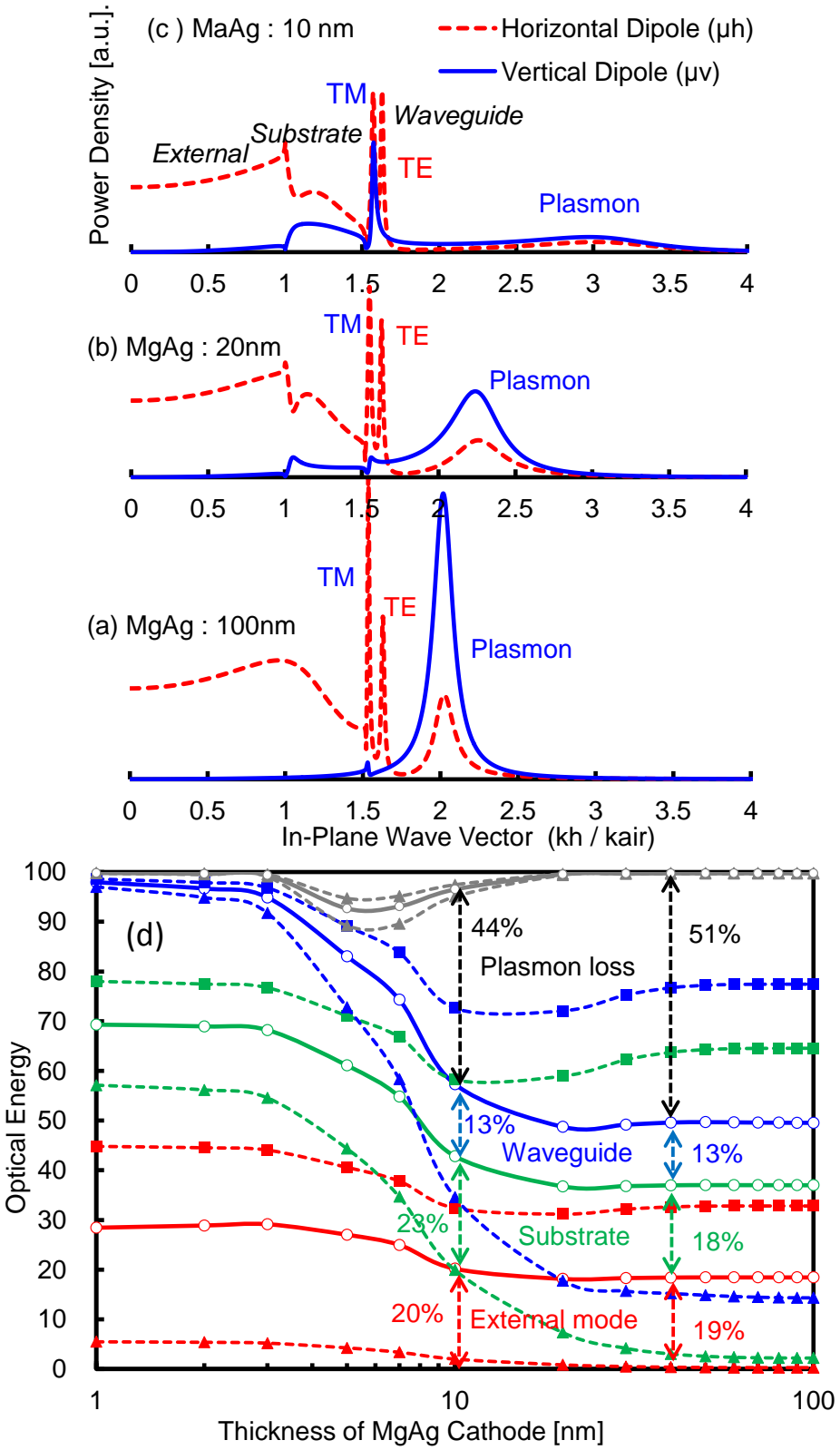

Figure 2. Optical power spectra (a)-(c) and optical mode energy as a function of the thickness of MgAg cathode. Solid lines: random dipole (O), Dashed lines : horizontal dipole ( $\mathbf{\square})$ and vertical dipole $(\boldsymbol{\Delta})$. 
$100 \mathrm{~nm}, 20 \mathrm{~nm}$ and $10 \mathrm{~nm}$ in thickness. Here, the in-plane wave vector is normalized by a wave-vector in air, therefore it can be considered that the horizontal axis is refractive index of each material used in the device. When the film thickness of MgAg cathode is $100 \mathrm{~nm}$, a strong surface plasmon pole appears at 2.02 in in-plane $\mathrm{k}$-space. This SP-pole tends to shift to higher $\mathrm{k}_{\mathrm{h}}$-value with a spread of half-width as the thickness of MgAg cathode is decreased to $20 \mathrm{~nm}$ and $10 \mathrm{~nm}$. A variation of optical power ratio with MgAg thickness is shown for horizontal, vertical and random dipoles in Figure 2(d). The SP-losses are $51 \%$ and $44 \%$ when the thicknesses of MgAg layer are $100 \mathrm{~nm}$ and $10 \mathrm{~nm}$, respectively. Although the SP-peak intensity actually becomes weaker with the decrease of MgAg thickness, total integration of the SP-loss hardly changes because k-space is expanded with the increase of wave-vector. Of course, the SP-loss becomes quickly small if the thickness is less than 10 $\mathrm{nm}$, we can't use such very thin film as an electrode, considering a lack of conductivity and uniformity. This clearly means that the SP-loss can be not suppressed even if it makes the cathode thickness much thinner.

The following equation is an actual reflection coefficient $\left(\mathrm{r}_{123}\right)$ in vertical dipole emission at the interface between ETL and MgAg/air, which is a complex number consisting of real and imaginary parts.

$$
r_{123}=\frac{r_{12}+r_{23} \exp \left(2 i k_{Z 2} t\right)}{1+r_{12} r_{23} \exp \left(2 i k_{Z 2} t\right)}=\operatorname{Re}\left(r_{123}\right)+\operatorname{Im}\left(r_{123}\right)=\left|r_{123}\right| \exp (-i \phi)
$$

Here $r_{12}$ and $r_{23}$ are reflection coefficients at the upper and lower interfaces of MgAg layer in the device structure shown in Figure 1. Symbol $\mathrm{t}$ is the thickness of $\mathrm{MgAg}$ layer, $\mathrm{k}_{\mathrm{Z} 2}$ is a wave vector in the normal direction and $\varphi$ is a phase angle. Imaginary part $\operatorname{Im}\left(\mathrm{r}_{123}\right)$ and phase $\phi$ are plotted as a function of in-plane wave vector in Figure 3. It is found that both the wave-vector and the intensity of SP-coupling shown in Figure 2 are directly related to Im ( $\left.\mathrm{r}_{123}\right)$ in this figure (a). Furthermore, it should be noted that the change of $\mathrm{k}_{\mathrm{h}}$ in SP-pole is strictly in agreement with the phase shift $\phi$. This results suggests that the SP-loss can be reduced if the phase shift in SP-coupling is driven out of the possible SP coupling region in k-space.

It is well known that penetration length of evanescent wave is an order of one wavelength.From the detail analysis, it turns out that the shift of in-plane wave vector of SP-peak is caused by the interaction between two kinds of SP-coupling on both sides of MgAg layer, when the film thickness of MgAg becomes thin so that an evanescent wave of dipole emission reaches the opposite side of MgAg/ETL layer. This means that plasmon resonance can be controlled by introducing rear material on backside of MgAg thin film.

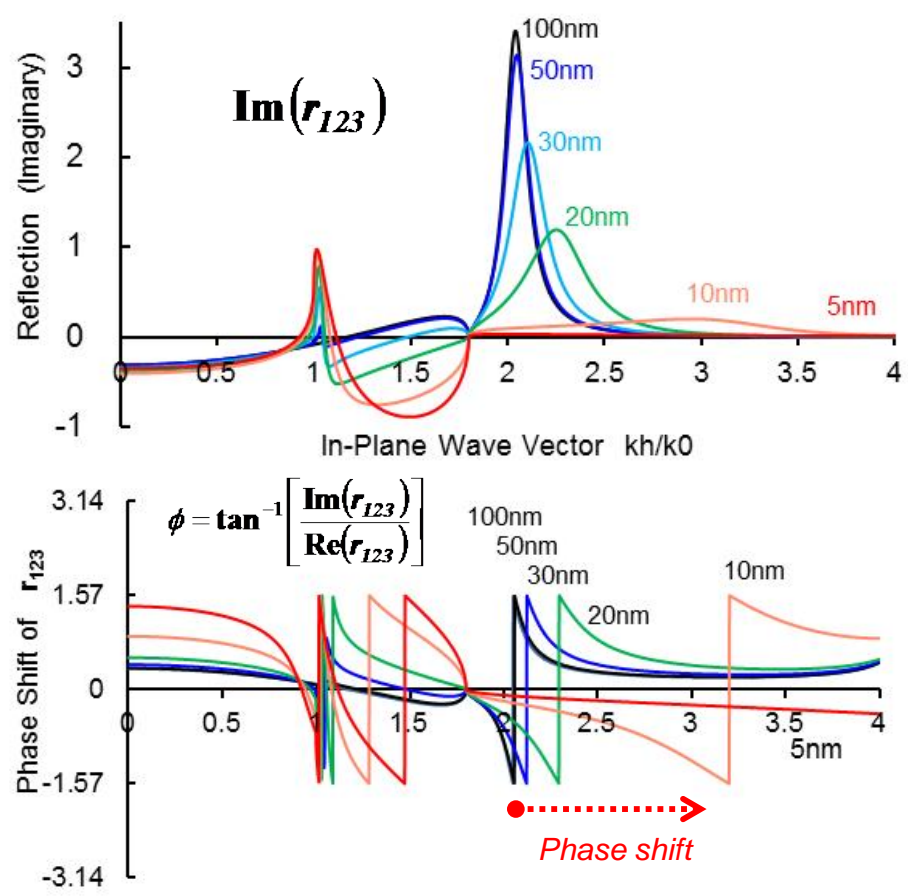

Figure 3. Variations of imaginary part $\operatorname{Im}\left(\mathrm{r}_{123}\right)$ and phase angle $\varphi$ of reflection coefficient at the interface between ETL and MgAg/Air with in-plane wave vector in OLED without OC-layer. 


\subsection{Control of Surface Plasmon Coupling by Optical Compensation}

We tried to deposit organic NPB film as an OC-layer on the back side of very thin MgAg cathode according to the previous consideration. Figures 4(a)-(c) show a power spectrum of electric dipole radiation without and with the OC-layer of $20 \mathrm{~nm}, 80 \mathrm{~nm}$ in thickness. As is expected, the power spectrum drastically changes with the thickness of OC-layer, and the SP-loss significantly becomes small at only $20 \mathrm{~nm}$ of OB-layer, and almost disappears at $80 \mathrm{~nm}$. Instead TM line grows greatly as a waveguide mode. Total integration of SP-loss was
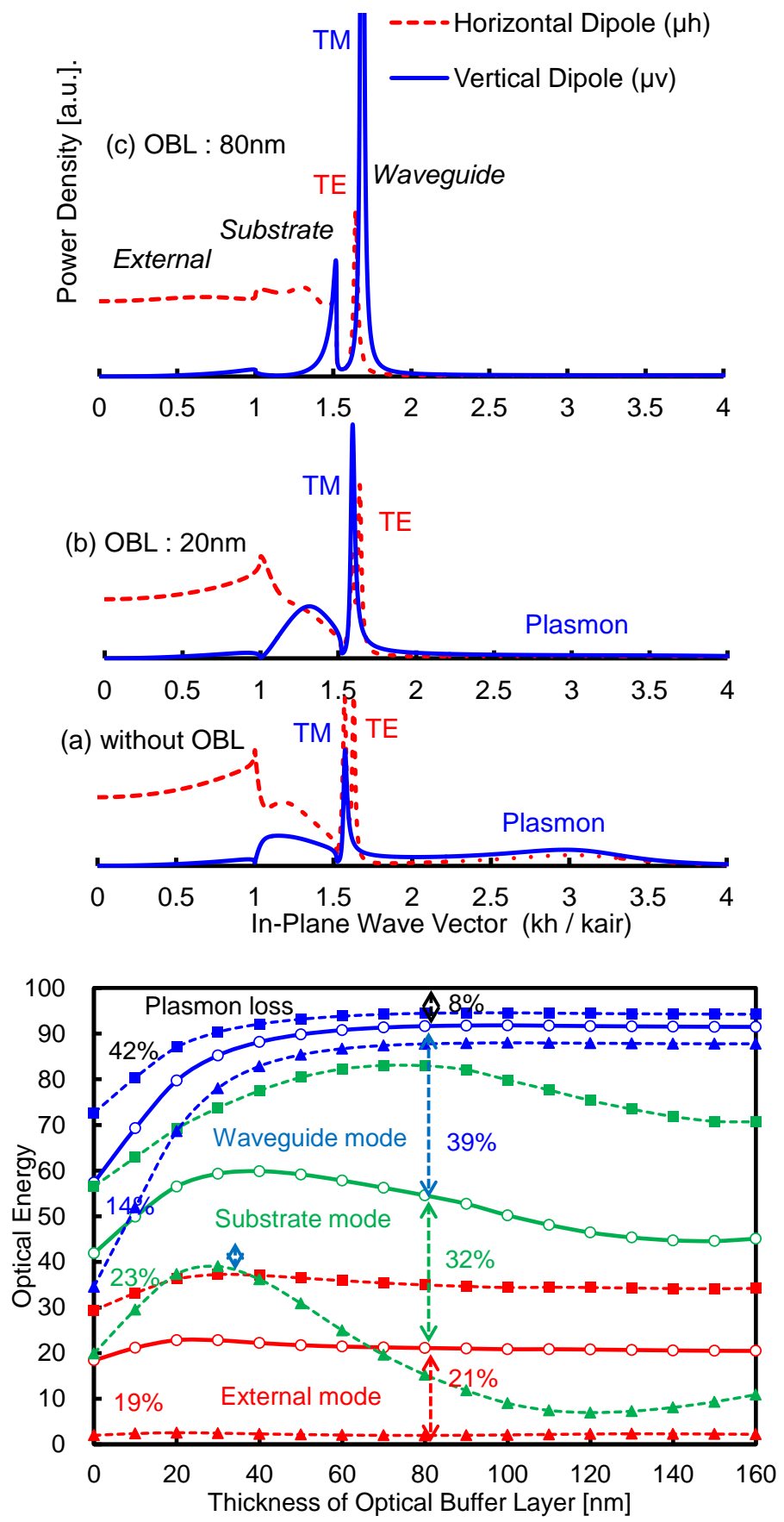

(d)

Figure 4. Power spectra and optical mode energy as a function of the thickness of optical compensation layer. The MgAg cathode is $10 \mathrm{~nm}$ in thickness. Marks and lines are the same as Figure 2. 
shown in figure (d) together with other propagation modes. The SP-loss becomes less than $10 \%$ at the OC-layer of above $80 \mathrm{~nm}$ in thickness. Waveguide and substrate modes gradually increase instead. The suppression of SPloss can be also possible not only the control of refractive index of the OC-layer but also the thickness of OClayer, namely it depends on the optical thickness of the OC-layer. Detail optical analysis clarified that the phase change of reflection coefficient on the interface of MgAg cathode makes the wave-vector of SP mode shifted to higher side beyond the limitation of plasma frequency. These results mean that the evanescent wave is successfully converted to the propagation wave by employing the OC-layer coupled with semi-transparent MgAg cathode. More than $90 \%$ of optical energy in dipole emission can be utilized as a propagation wave.

Figure 5 shows the change of power spectra in the wave-vector around the SP mode with the thickness of MgAg layer $\left(\mathrm{d}_{\mathrm{MgAg}}\right)$. The thickness of the OC layer was kept constant of $120 \mathrm{~nm}$. When $\mathrm{d}_{\mathrm{MgAg}}$ is $100 \mathrm{~nm}$, a strong SP pole appears at 2.02 as well as the normal cathode structure. However, when $\mathrm{d}_{\mathrm{MgAg}}$ is less than $60 \mathrm{~nm}$, the SP pole is divided to two peaks, which are corresponding to long range SP mode (LRSP) in the lower wave-vector and short range SP mode (SRSP) in the higher wave-vector, respectively. As $\mathrm{d}_{\mathrm{MgAg}}$ is further decreased less than $20 \mathrm{~nm}$, LRSP peak becomes together with waveguide mode, and changes to a propagation wave. On the other hand, since the wave vector of SRSP exceeds the limit of resonance, it disappears. As a result, almost all of the evanescent wave can be successfully converted to propagation wave.

\subsection{Device Performance}

We have experimentally prepared a phosphorescent green OLED with MLC structure shown in Figure 1(b) and compared the emission efficiency with the normal OLED shown in in Figure 1(a) in order to make sure of the reduced SP-loss. Figure 6 shows an external quantum efficiency (EQE) of three OLEDs with the normal cathode (Device A) and MLC structure. Devices (Device B and C). Refractive index of the glass substrate are 1.52 for device A and B, and 1.80 for device $\mathrm{C}$, because high refractive index glass has an effect of increasing substrate mode [6]. In addition, the micro-lens sheet for taking out substrate mode outside is stuck on the back of all the substrate glass. The maximum efficiencies in EQE are $23 \%, 46 \%$ and $64 \%$ in the devices a, B and C, respectively. EQE has surely improved about twice in MLC structure.

It is possible to visualize the behavior of propagation light intensity by using FDTD simulation. Figures 6(a)-(c) show optical power density distribution in the devices A, B and C, respectively. Since the SP loss mainly originates in vertical dipole, the calculation of electric field intensity in layers was carried out only by exciting the vertical dipole moment at the interface between EML and ETL. Optical power intensity in the waveguide mode can be clearly increased in the device B and C with MLC structure compared with the device A with the normal cathode. Furthermore, by using high refractive-index glass, it turns out that the waveguide mode is moving to the substrate as shown in Figure 6(c).

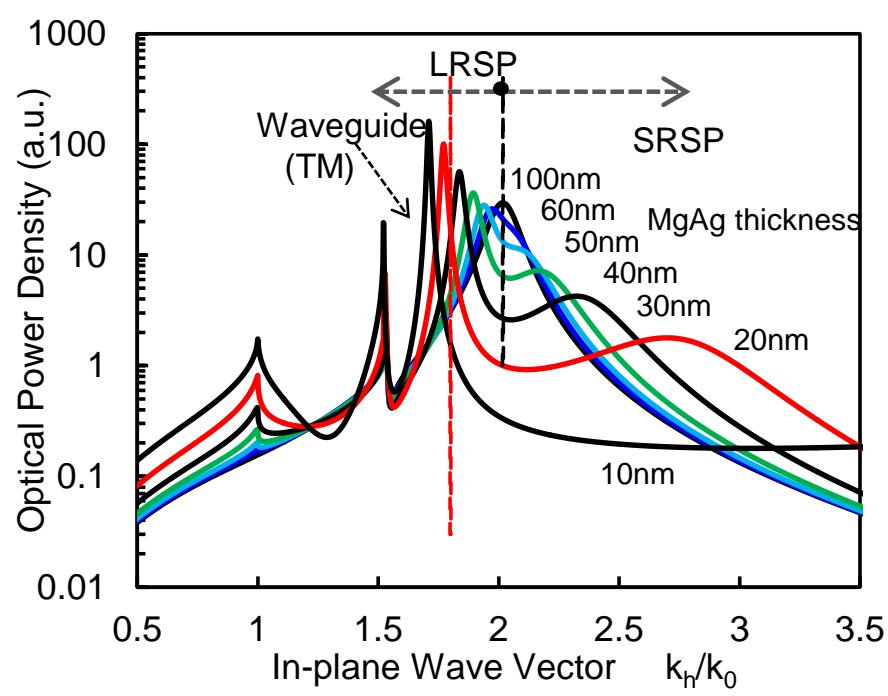

Figure 5. Variation of optical power spectra with the thickness of MgAg layer. Long range (LRSP) and short range (SRSP) surface plasmon sift in the opposite direction mutually as the MgAg thickness is decreased. 

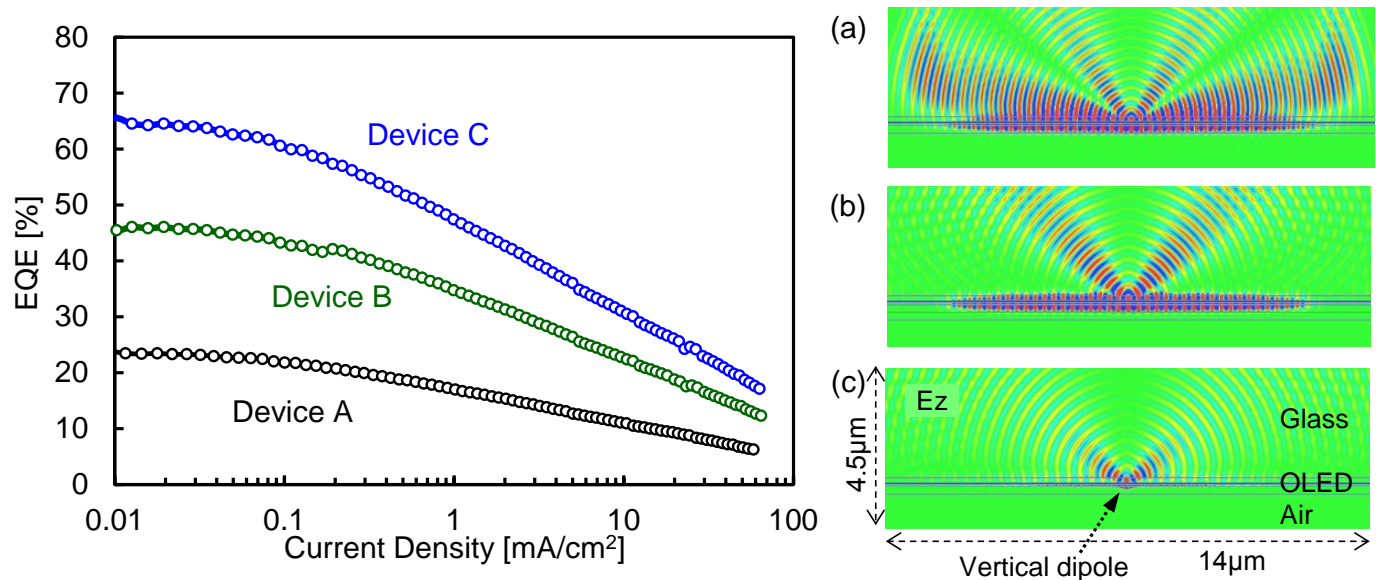

Figure 6. External quantum efficiency vs. current density characteristics in OLEDs with the normal structure (Device A), this work's device with MLC structure (Devices B and C). Refractive indexes of glass substrate are 1.52 for the devices A and B, 1.80 for the device C. The micro-lens sheet is stuck on the back of all the glass substrate. (a)-(c) are electric field intensity of propagation light in each layer induced by vertical dipole on the light emitting layer in OLEDs.

\section{Conclusion}

The effect of reducing a SP-loss was investigated in OLED with a nano-sized MLC structure. In results, huge loss due to SP coupling with metal cathode was converted to propagation wave such as waveguide and substrate modes. These phenomena can be explained by the change of wave vector in SP-coupling induced by the interaction of two SP modes (LRSP and SRSP) between both interfaces of MgAg layer. We would like to emphasize that this technique is effective for the improvement of the light extraction efficiency in OLEDs. In addition, we would like to emphasize that multi-scale optical analysis including near-field optics is useful for the optical design of high efficiency OLED application.

\section{Acknowledgements}

This research activity was supported by a Grant-in-Aid for scientific research No. 25410252 from the Japan Society for the Promotion of Science. Authors want to acknowledge the funding received from that.

\section{References}

[1] Baldo, M.A., Lamansky, S., Burrow, P.E., Thompson, M.E. and Forrest, S.R. (1999) Very High-Efficiency Green Organic Light-Emitting Devices Based on Electrophosphorescence. Applied Physics Letter, 75, 4-6. http://dx.doi.org/10.1063/1.124258

[2] Netzs, K.A. (1998) Simulation of Light Emission from Thin-Film Microcavities, Journal of Optical Society of America A, 15, 962-971. http://dx.doi.org/10.1364/JOSAA.15.000962

[3] Mikami, A. and Hitoshi, I. (2014) Optical Control of Surface Plasmon Coupling in Organic Light Emitting Devices with Nanosized Multi-cathode Structure. Proceedings of the 5th International Conference on Nanotechnology, Czech Republic, 11-13 August 2014.

[4] Mikami, A. (2013) Optical Design of Enhanced Light Extraction Efficiency in Organic Light Emitting Devices with an Optically Controlled Surface Plasmon Coupling. Journal of Light \& Environment, 37, 26-29. http://dx.doi.org/10.2150/jlve.ieij130000502

[5] Ford, G.E. and Wever, W.H. (1984) Electromagnetic Interaction of Molecules with Metal Structure. Physics Report 113, No. 4, 196-287, North-Holland, Amsterdam. http://dx.doi.org/10.1016/0370-1573(84)90098-X

[6] Mikami, A. (2013) Optical Design of 200-lm/W Phosphorescent Green Light Emitting Devices Based on the High Refractive Index Substrate. Physica Status Solidi, C8, 2899-2902. http://dx.doi.org/10.1002/pssc.201084041 


\section{Submit or recommend next manuscript to SCIRP and we will provide best service for you:}

Accepting pre-submission inquiries through Email, Facebook, LinkedIn, Twitter, etc.

A wide selection of journals (inclusive of 9 subjects, more than 200 journals)

Providing 24-hour high-quality service

User-friendly online submission system

Fair and swift peer-review system

Efficient typesetting and proofreading procedure

Display of the result of downloads and visits, as well as the number of cited articles

Maximum dissemination of your research work

Submit your manuscript at: http://papersubmission.scirp.org/ 